\title{
OPEN Effect of 4-week physical exercises on tryptophan, kynurenine and kynurenic acid content in human sweat
}

\author{
Tomasz Saran $\circledast^{1}$, Monika Turska $\mathbb{1}^{2}$, Tomasz Kocki $\mathbb{1}^{3}$, Magdalena Zawadka $\mathbb{1}^{4}$, \\ Grzegorz Zieliński ${ }^{4 \bowtie}$, Waldemar A. Turski $\mathbb{1}^{3}$ \& Piotr Gawda $\mathbb{1}^{4}$
}

The aim of the study was the detection of TRP, kynurenine (KYN), and kynurenic acid (KYNA) in human sweat, and determining whether physical activity affects their content in this secrete. Two different methods were used simultaneously-collection of sweat by means of an absorption pad from the inter scapular region, and collection of a drop of sweat from the region of the forehead. Quantitative determinations of TRP, KYN and KYNA were performed using high performance liquid chromatography with ultraviolet and fluorescence detection. Determinations of sodium was carried out by the method of inductively coupled plasma collision/reaction cell ionization mass spectrophotometry. It was found that physical exercises evoked a decrease in the amount of KYN, and an increase in the amount of KYNA in sweat recorded on day 14, but not on day 28 of training. It appears that physical exercises result in a long-term increase in the kynurenine transaminase activity responsible for the formation of KYNA from KYN. Based on this results, it can be suggested that measurement of TRP, KYN and KYNA in sweat may have diagnostic potential and may help to establish an exercise regime appropriate for the age, gender and health status of rehabilitation patients.

Kynurenine (KYN) is a primary metabolite of tryptophan (TRP) formed along the kynurenine pathway. In mammals, the reaction is catalyzed by tryptophan-2,3-dioxygenase (TDO) or indoleamine-2,3-dioxygenases 1 and 2 (IDO). TDO is a constitutive enzyme present primarily in the liver. Its activity is controlled by available TRP, circulating glucocorticoid and heme while feedback inhibition is exerted by NAD(P)H. IDO 1 is an inducible enzyme expressed in almost all extrahepatic cells the activity of which is raised by cytokines during immune activation. Interferon- $\gamma$ is thought to be a principal effector and nitric oxide is an inhibitor. The role of IDO2 is less known. In comparison to IDO1 it catalyzes TRP degradation under similar condition, but far less efficiently ${ }^{1,2}$. KYN is further metabolized to 3-hydroxykynurenine by kynurenine monoxygenase, anthranilic acid by kynureninase and kynurenic acid (KYNA) by kynurenine aminotransferases ${ }^{2}$. Subsequent bioactive metabolites formed along kynurenine pathway are 3-hydroxyantranilic acid, quinolinic acid and finally nicotinamide adenine dinucleotide ${ }^{2}$.

KYN and its metabolites, collectively called kynurenines, possess diverse biological activities. The role of kynurenines in the central nervous system is a subject of intensive research ${ }^{3,4}$ however, in skeletal muscle is less well understood and remains largely unknown. It was discovered only recently that an intensive transformation of KYN to KYNA takes place in exercising skeletal muscle ${ }^{5-8}$. Currently, it is widely accepted that physical training may exert its beneficial effect on the central nervous system due to the reduction of circulating KYN available for further metabolism in the brain ${ }^{5,9,10}$. KYN is an agonist of aryl hydrocarbon receptor $(\mathrm{AhR})^{11}$. KYNA is an antagonist of glutamate receptors, acting predominantly on $\mathrm{N}$-methyl-D-aspartate (NMDA) receptor. It is also an antagonist of $\alpha 7$-nicotinic acetylcholine receptor and agonist of $\mathrm{G}$ protein-coupled receptor 35 (GPR-35) and $\mathrm{AhR}^{12}$. The potential role of kynurenines in pathophysiology of the central nervous system has been extensively investigated for more than 3 decades. Initially, it was evidenced that both quinolinic acid and KYNA, glutamate

${ }^{1}$ Department of General and Neurorehabilitation, Institute of Rural Health in Lublin, Lublin, Poland. ${ }^{2}$ Department of Pharmacology, Faculty of Health Sciences, Medical University of Lublin, Lublin, Poland. ${ }^{3}$ Department of Experimental and Clinical Pharmacology, Medical University of Lublin, Lublin, Poland. ${ }^{4}$ Department of Sports Medicine, Faculty of Health Sciences, Medical University of Lublin, Lublin, Poland. ${ }^{\circledR}$ email: grzegorz.zielinski@ umlub.pl 
agonist and antagonist, respectively, are involved in the control of neurotransmission, neuronal membrane excitability and neurodegeneration. Afterwards, evidence for association of imbalanced kynurenine pathway with neurological and psychiatric disorders was accumulated. Nowadays, the participation of KYN metabolites in psychoneuroimmunological conditions is advocated ${ }^{13-15}$. Thus, the link between exercise-induced changes in peripheral kynurenine homeostasis and brain functioning appears indisputable ${ }^{10,16,17}$.

KYNA is end metabolite which is excreted mainly with urine ${ }^{18}$. Nevertheless, elevated content of KYNA in plasma may have biological consequences. Since KYNA poorly penetrate the blood-brain barrier ${ }^{19}$ it is unlikely that it affects functioning of the central nervous system. On the contrary, peripheral administration of KYNA exerted antiulcerative activity ${ }^{20}$, reduced inflammation and modulated motility and contractility of the colon ${ }^{21,22}$, increased renal excretion ${ }^{23}$, reduced heart rate ${ }^{24}$ and exerted a cardioprotective effect ${ }^{25}$. Subsequent bioactive metabolites formed along kynurenine pathway are 3-hydroxyantranilic acid, quinolinic acid and finally nicotinamide adenine dinucleotide ${ }^{2}$.

To date, research focused on the effect of physical exercises on kynurenine pathway activity, is based mainly on the measurement of the content of kynurenines and enzymes of the kynurenine pathway in the plasma or tissue collected by means of muscle biopsy ${ }^{26,27}$. Both biopsy and blood sampling are invasive methods and require professional staff and aseptic conditions. As yet, there have been no reports on monitoring the effects of exercise on the level of kynurenines with the use of non-invasive methods.

Since kynurenines were found in secretes such as saliva ${ }^{28,29}$ and human milk ${ }^{30,31}$ it is assumed that they can also be present in sweat. Therefore, the aim of this study was to assess whether TRP, KYN and KYNA are present in a measurable amount in sweat, and to establish the dynamic of changes of their concentrations in human sweat during a 4-week specific physical training in previously untrained individuals.

\section{Methods}

This study was performed in line with the principles of the Helsinki Declaration. Approval was granted by the Ethics Committee at the Institute of Rural Health in Lublin, Poland (Approval No. 3/2015). Study participants were recruited from patients of the Department of General and Neuro-Rehabilitation. The following criteria of inclusion were applied: males and females aged 25-65 years, persons with chronic ( $>3$ months) mild to moderate low back pain, capable of undertaking moderate endurance training. Eligible patients were informed of the study during their first visit to the out-patient clinic and signed an informed consent before any study-related procedures were performed. A health interview and medical examination were performed before inclusion to the study group. The following exclusion criteria were applied: impaired mobility of joints, which would make it difficult to exercise on devices, permanent or periodic intake of anti-inflammatory and analgesic drugs, duration of ailments less for than four weeks, night or continuous back pain, the presence of sensory symptoms or motor spinal cord injury, systemic inflammatory diseases, obesity, glucose intolerance or diabetes, impaired renal function, heart failure and ischemic heart disease, decompensated hypertension or treatment with diuretics, chronic central nervous system disorders, including mental diseases and history of alcohol or substance abuse, physical or mental disabilities precluding physical testing and/or exercise, and inability to read and understand Polish. A screening instrument was applied to control the state of psychological health-the General Health Questionnaire GHQ-28 D. Goldberg polish adaptation. Only patients with a score lower than 5 were included in the study ${ }^{32}$.

Due to the dietary origin of the pool of TRP, and the crucial involvement of gut microbiota in kynurenine pathway regulation $s^{17}$ participants were instructed to continue their eating habits and to inform the investigator about probiotics and medication use throughout the period of the study; however, there were no such reports among participants.

The study group consisted of 35 Caucasian women $(n=25)$ and men $(n=10)$, who did not engage in regular physical activity. The mean age was 47.62 years $(S D \pm 15.91)$ and the mean Body Mass Index (BMI) was $24.36 \mathrm{~kg} /$ $\mathrm{m}^{2}(\mathrm{SD} \pm 3.29)$.

The study participants performed the daily exercise under medical supervision every morning for four consecutive weeks (except Saturdays and Sundays). The two-phase training programme consisted of exercises on a bicycle cycloergometer, followed by exercises on the elliptical cross-trainer. In the first week, the training on each device lasted eight minutes, in the second week it was extended to $10 \mathrm{~min}$, whereas in the third and fourth weeks it took $15 \mathrm{~min}$. There were no breaks longer than $30 \mathrm{~s}$ between exercises on both devices. Intensity of the exercise was submaximal. The maximum heart rate was calculated according to the formula: $208-0.7 \times$ age in years. $85 \%$ of the maximum heart rate value was assumed as the submaximal value. The exercises were performed at a temperature of $23-24^{\circ} \mathrm{C}$ in an air-conditioned gymnasium.

Sweat samples were collected on days one, 14 and 28 of the training. Due to possible errors arising from the specificity of a sweat sampling method, in the present study two different methods of collecting sweat samples were applied: Method A-a commercial absorbent patch (Pharm Chem Inc., USA), and Method B-a cotton bud of commercial ear-tips (Bella Cotton, Poland) were used. Method A: before starting the training session a PharmCheck patch was placed on the skin between the shoulder blades. The patch was removed immediately after finishing the daily training. Method B: a sample (a drop of sweat) was collected from the patient's forehead with a cotton wool swab immediately after finishing the daily training. Next, the pads from patches and the swabs were placed in polyethylene tubes and frozen at $-25^{\circ} \mathrm{C}$. After defrosting, $0.5 \mathrm{ml}$ of de-ionized water was added. The solution was separated from pads and swabs by means of centrifugation $\left(4^{\circ} \mathrm{C}, 15 \mathrm{~min}, 15,000 \mathrm{rpm}\right)$ and collected for analyses.

Determinations of plasma sodium were made with a ThermoScientific model XSERIES2 mass spectrometer (USA), with a collision-collision reaction chamber, with ionisation in inductively coupled plasma (ICP-MS). Parameters of the measurement system: nebulizer-glass concentric; spraychamber-glassconical impact bead; Interface option-Xt; sample uptake rate $-0.4 \mathrm{~mL} / \mathrm{min}$, approx. pumped; quadrupole resolution - standard 
resolution peak width $0.70 \mathrm{amu}$ at $5 \%$ height; plasma gas-Argon (5 grade) $15 \mathrm{~L} / \mathrm{min}$; plasma gas CCT-8\% Hydrogen in Helium (5 grade) $5 \mathrm{ml} / \mathrm{min}$; sample uptake time-15 s; wash delay-35 s; total time per sample-3 min, $5 \mathrm{~s}$; number of replicates per sample-3. Reagents of purity for trace analysis and deionized water purified with the Millipore Simplicity 185 UV apparatus (USA) were used. The Inorganic Ventures ANALITYK122 (USA) pattern was used for calibration of the spectrometer. For the correctness of the calibration curves and for quality control of the performed analyzes, the following certified reference materials were used: EnviroMat ES-L-2 and EnviroMat ES-H-2.

TRP, KYN, and KYNA were measured with high-performance liquid chromatography (HPLC) according to Zhao et al. 2010 with modifications ${ }^{33}$. The HPLC equipment consisted of UltiMate 3000 analytical HPLC system with UltiMate 3000 Autosampler with thermostated column compartment (Thermo Fisher Scientific, Waltham, $\mathrm{MA}$, United States). The samples were kept at a constant temperature $\left(4.0^{\circ} \mathrm{C}\right)$ during the analysis. Chromatography column was set at $30^{\circ} \mathrm{C}$. The HPLC system was equipped with an analytical column (Agilent HC-C18 (2) column; $250 \times 4.6 \mathrm{~mm}$ i.d.; $5 \mu \mathrm{m}$ particle size. The mobile phase consisted of $20 \mathrm{mmol} / \mathrm{L}$ sodium acetate, $3 \mathrm{mmol} / \mathrm{L}$ zinc acetate, and $7 \%$ acetonitrile; flow-rate was set at $1 \mathrm{~mL} / \mathrm{min}$. All reagents were provided by J. T. Baker and Sigma-Aldrich. The samples were deproteinized with $6 \%$ perchloric acid and then centrifuged (30 min, $15,000 \mathrm{rpm}$ ). The volume per sample injection was $100 \mu \mathrm{L}$. KYN and TRP were measured by UV detection (set for $365 \mathrm{~nm}$ and $250 \mathrm{~nm}$, respectively). KYNA was analyzed fluorometrically (excitation $344 \mathrm{~nm}$, emission $398 \mathrm{~nm}$ ). A Chromeleon software was employed to control the HPLC system and process the chromatographic data.

Data are presented as the content of a determined substance in fmol per $\mu \mathrm{g}$ of $\mathrm{Na}$. Activity of enzymes IDO\&TDO and KAT was defined as the ratio of KYN/TRP $\times 100$ and KYNA/KYN, respectively ${ }^{34}$. Data concerning age and BMI of participants are presented as a mean \pm standard deviation (SD). The content of sodium, substances in sweat and calculated enzyme activity were expressed as the median with a range of variation (25-75\%). Statistical analysis was conducted using Statistica software (ver. 13.1). The statistical comparisons of results were performed using an analysis of variance (ANOVA) for dependent samples with type I error set at $5 \%$ and type II error set at $20 \%$ ( $80 \%$ power). We determined the minimum number of subjects for adequate study power. The required sample size resulted in $\mathrm{N}=26$.

The distribution of data was verified using the Shapiro-Wilk test. Intra-group comparisons for normally distributed data were performed using Student's $t$ test for dependent samples. For non-normally distributed data, analysis was performed using a non-parametric Wilcoxon rank test. Correlation analyzes, after verifying the linearity, were carried out using the non-parametric Spearman rho correlation coefficient. Statistical significance was accepted at $\mathrm{p}<0.05$.

\section{Results}

The amounts of $\mathrm{Na}$ in the samples collected by the Method A and B on days 14 and 28 did not significantly differ from the value obtained on day one (Table 1).

The amounts of TRP in the sweat samples collected by Method A and B on days 14 and 28 did not differ statistically from the values obtained on day one. The content of KYN in sweat samples collected by method A and B on day 14 but not 28 was significantly reduced in comparison to respective control value. The amount of KYNA in the sweat samples collected by Method A and B on day 14 but not 28 was significantly elevated in comparison to respective control value (Table 1).

The activity of KAT in the sweat samples collected by Method A and B on day 14 and 28 was significantly elevated, compared to respective control values obtained on day one. IDO\&TDO activity in samples collected by Method A and B on day 14 and 28 was significantly reduced in comparison to control values obtained on day one (Table 1).

\section{Discussion}

The study shows for the first time that metabolites of the kynurenine pathway, KYN, and KYNA, are present in human sweat. TRP was also found in human sweat, although its existence in this secretion was recently announced in a metabolomic study by Cui et al. ${ }^{35}$. It is noteworthy that the current study showed that content of KYN and KYNA varies depending on physical activity, while the amount of TRP in sweat remained unchanged. Interestingly, a large increase in KYNA and significant reduction of KYN content in human sweat was found on day 14 on the physical exercise cycle. On day 28 , the differences were insignificant. These findings suggest that physical activity affects kynurenine pathway activity. Therefore, the activity of enzymes responsible for transformation of TRP to KYN and KYN to KYNA were assessed. Enzyme activity was calculated as a ratio of product-to-precursor content. This revealed that IDO\&TDO activity was decreased and KAT activity increased throughout the entire exercise cycle. As far as sweat composition reflects the content of substances in blood and tissues, the obtained results point to the conclusion that physical activity leads to the reduction of KYN at the expense of KYNA synthesis.

Saliva seems to be another attractive source of biological material which can be obtained non-invasively. However, it should be considered that presence of KYNA synthetizing enzymes KAT I-III in human saliva and production of KYNA by saliva has been described recently ${ }^{36}$. The stability of kynurenines in such material seems to be a problematic issue. Thus, the usability of saliva for determination of KYNA and other kynurenines as a reflection of their content in the body should be experimentally confirmed.

The results of previous trials in humans studying the effect of physical activity on the kynurenine pathway metabolites agree with the findings of the current study. It should be stressed, however, that in all of the studies the content of kynurenine metabolites formed along the kynurenine pathway was measured in blood. By testing plasma composition in 28 competitors before and after the Boston Marathon, Lewis et al. found an increase of KYNA up to $189 \%$ and reduction of TRP up to $23 \%$ of initial values ${ }^{37}$. Mudry et al. reported that one-time 


\begin{tabular}{|c|c|c|c|c|c|c|}
\hline & & \multirow[b]{2}{*}{1 day } & \multirow[b]{2}{*}{14 day } & \multirow[b]{2}{*}{28 day } & \multicolumn{2}{|l|}{ Significance } \\
\hline & & & & & $\begin{array}{l}14 \text { day } \\
\text { vs. } 1 \text { day }\end{array}$ & $\begin{array}{l}28 \text { day } \\
\text { vs. } 1 \text { day }\end{array}$ \\
\hline \multirow{6}{*}{ Method A } & $\mathrm{Na}[\mu \mathrm{g} / \mathrm{sample}]$ & \begin{tabular}{|l|}
183.77 \\
$(19.91-529.45)$
\end{tabular} & $214.65(152.52-320.36)$ & $204.89(100.66-375.46)$ & $\begin{array}{l}Z=0.57 \\
p>0.05\end{array}$ & $\begin{array}{l}Z=0.37 \\
p>0.05\end{array}$ \\
\hline & $\begin{array}{l}\text { TRP } \\
{[\mathrm{fmol} / \mu \mathrm{g} \mathrm{Na}]}\end{array}$ & $\begin{array}{l}586.00 \\
(245.11-1132.98)\end{array}$ & $\begin{array}{l}763.76(549.60- \\
1259.84)\end{array}$ & $892.38(399.25-1668.04$ & $\begin{array}{l}Z=0.78 \\
p>0.05\end{array}$ & $\begin{array}{l}Z=1.58 \\
p>0.05\end{array}$ \\
\hline & $\begin{array}{l}\mathrm{KYN} \\
{[\mathrm{fmol} / \mu \mathrm{g} \mathrm{Na}]}\end{array}$ & $\begin{array}{l}73.93 \\
(16.47-280.16)\end{array}$ & \begin{tabular}{|l|}
7.44 \\
$(3.47-16.09)$
\end{tabular} & $\begin{array}{l}15.27 \\
(7.31-52.42)\end{array}$ & $\begin{array}{l}Z=3.07 \\
\mathbf{p}<\mathbf{0 . 0 1}\end{array}$ & $\begin{array}{l}Z=0.80 \\
p>0.05\end{array}$ \\
\hline & \begin{tabular}{|l|}
$\mathrm{KYNA}$ \\
{$[\mathrm{fmol} / \mu \mathrm{g} \mathrm{Na}]$}
\end{tabular} & $\begin{array}{l}14.80 \\
(1.30-54.58)\end{array}$ & \begin{tabular}{|l|}
55.64 \\
$(27.58-113.81)$
\end{tabular} & $\begin{array}{l}15.26 \\
(8.58-50.01)\end{array}$ & $\begin{array}{l}Z=1.29 \\
\mathbf{p}<\mathbf{0 . 0 5}\end{array}$ & $\begin{array}{l}Z=0.24 \\
p>0.05\end{array}$ \\
\hline & KAT & \begin{tabular}{|l|}
0.12 \\
$(0.05-0.15)$
\end{tabular} & \begin{tabular}{|l|}
6.62 \\
$(4.15-8.52)$
\end{tabular} & $\begin{array}{l}1.47 \\
(0.37-2.32)\end{array}$ & $Z=3.92 \mathbf{p}<\mathbf{0 . 0 0 1}$ & $\begin{array}{l}Z=2.93 \\
\mathbf{p}<\mathbf{0 . 0 1}\end{array}$ \\
\hline & IDO/TDO & $\begin{array}{l}53.31 \\
(7.75-112.45)\end{array}$ & \begin{tabular}{|l|}
0.92 \\
$(0.80-1.20)$
\end{tabular} & $\begin{array}{l}2.91 \\
(2.16-4.45)\end{array}$ & $\mathrm{Z}=4.01 \mathbf{p}<\mathbf{0 . 0 0 1}$ & $\begin{array}{l}Z=3.27 \\
\mathbf{p}<\mathbf{0 . 0 5}\end{array}$ \\
\hline \multirow{6}{*}{ Method B } & $\mathrm{Na}[\mu \mathrm{g} / \mathrm{sample}]$ & $164.30(28.75-1136.33)$ & $\begin{array}{l}114.98 \\
(47.60-285.93)\end{array}$ & $\begin{array}{l}206.53 \\
(57.20-552.80)\end{array}$ & $\begin{array}{l}Z=1.25 \\
p>0.05\end{array}$ & $\begin{array}{l}Z=1.53 \\
p>0.05\end{array}$ \\
\hline & \begin{tabular}{|l|} 
TRP \\
{$[\mathrm{fmol} / \mu \mathrm{g} \mathrm{Na}]$}
\end{tabular} & $\begin{array}{l}448.30(283.90- \\
1717.62)\end{array}$ & \begin{tabular}{|l|}
925.52 \\
$(694.83-3267.29)$
\end{tabular} & $\begin{array}{l}473.68(129.45- \\
8182.81)\end{array}$ & $\begin{array}{l}Z=1.59 \\
p>0.05\end{array}$ & $\begin{array}{l}Z=1.01 \\
p>0.05\end{array}$ \\
\hline & $\begin{array}{l}\mathrm{KYN} \\
{[\mathrm{fmol} / \mu \mathrm{g} \mathrm{Na}]}\end{array}$ & $\begin{array}{l}34.27 \\
(15.16-677.08)\end{array}$ & $\begin{array}{l}11.87 \\
(7.44-40.68)\end{array}$ & $\begin{array}{l}15.47 \\
(7.10-103.24)\end{array}$ & $\begin{array}{l}Z=2.44 \\
\mathbf{p}<\mathbf{0 . 0 5}\end{array}$ & $\begin{array}{l}Z=0.31 \\
p>0.05\end{array}$ \\
\hline & $\begin{array}{l}\mathrm{KYNA} \\
{[\mathrm{fmol} / \mu \mathrm{g} \mathrm{Na}]}\end{array}$ & $\begin{array}{l}8.16 \\
(2.41-53.98)\end{array}$ & $\begin{array}{l}133.03 \\
(31.32-208.27)\end{array}$ & $\begin{array}{l}26.60 \\
(8.98-79.06)\end{array}$ & $\begin{array}{l}Z=2.56 \\
\mathbf{p}<\mathbf{0 . 0 5}\end{array}$ & $\begin{array}{l}Z=0.80 \\
p>0.05\end{array}$ \\
\hline & KAT & $\begin{array}{l}0.12 \\
(0.05-0.29)\end{array}$ & \begin{tabular}{|l|}
6.13 \\
$(4.11-8.39)$
\end{tabular} & \begin{tabular}{|l|}
0.81 \\
$(0.22-2.76)$
\end{tabular} & $\mathrm{Z}=4.07 \mathbf{p}<\mathbf{0 . 0 0 1}$ & $\begin{array}{l}Z=2.58 \\
\mathbf{p}<\mathbf{0 . 0 1}\end{array}$ \\
\hline & IDO/TDO & $\begin{array}{l}78.24 \\
(4.12-140.12)\end{array}$ & $\begin{array}{l}1.10 \\
(0.85-1.28)\end{array}$ & $\begin{array}{l}2.64 \\
(1.56-3.29)\end{array}$ & $\mathrm{Z}=3.84 \mathbf{p}<\mathbf{0 . 0 0 1}$ & $\begin{array}{l}\mathrm{Z}=2.59 \\
\mathbf{p}<\mathbf{0 . 0 1}\end{array}$ \\
\hline
\end{tabular}

Table 1. Content of natrium (Na), tryptophan (TRP), kynurenine (KYN) and kynurenic acid (KYNA), and activity of indoleamine-2,3-dioxygenase and tryptophan-2,3-dioxygenase (IDO\&TDO) and kynurenine aminotransferase (KAT) in human sweat during physical training: upper panel-sampling method A; lower panel-sampling method B. Data expressed as the median with a range of variation (25-75\%) are presented as the content of determined substance in fmol per $\mu \mathrm{g}$ of $\mathrm{Na}$.

\begin{tabular}{|c|c|c|c|c|c|}
\hline Physical activity & Time & Tryptophan & Kynurenine & Kynurenic acid & Literature \\
\hline \multirow{2}{*}{$\begin{array}{l}\text { Rugby camp for three days; bicycle ergometry, rugby } \\
\text { training, soccer game and } 20 \mathrm{~km} \text { run }\end{array}$} & post exercise & ND & $\uparrow$ & ND & \multirow{2}{*}{40} \\
\hline & $24 \mathrm{~h}$ after exercise & ND & $=$ & ND & \\
\hline \multirow{2}{*}{ Acute graded exercise test on bicycle ergometer } & post exercise & $\downarrow$ & $\downarrow$ & $\uparrow$ & \multirow{2}{*}{6} \\
\hline & $3 \mathrm{~h}$ after exercise & $\downarrow$ & $\downarrow$ & $\uparrow$ & \\
\hline Cycle ergometer exercise test for 12 weeks & 5 min after exercise & $\downarrow$ & $=$ & ND & 41 \\
\hline Exhaustive aerobic exercise & 5 min after exercise & $\downarrow$ & $\uparrow$ & ND & 42 \\
\hline \multirow{2}{*}{ Acute endurance exercise } & post exercise & $=$ & $=$ & $\uparrow$ & \multirow{4}{*}{34} \\
\hline & $1 \mathrm{~h}$ after exercise & $=$ & $=$ & $=$ & \\
\hline \multirow{2}{*}{ Resistance exercise } & post exercise & $\downarrow$ & $=$ & $\uparrow$ & \\
\hline & $1 \mathrm{~h}$ after exercise & $=$ & $=$ & $\downarrow$ & \\
\hline Half-marathon race & $30 \mathrm{~min}$ after race & ND & ND & $\uparrow$ & \multirow[t]{2}{*}{43} \\
\hline $150 \mathrm{~km}$ cycling & $1 \mathrm{~h}$ after cycling & ND & ND & 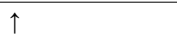 & \\
\hline
\end{tabular}

Table 2. Effects of physical activities on tryptophan, kynurenine and kynurenic acid content in human blood: an overview. $\uparrow$ increase; $\downarrow$ decrease; = no change; ND—no data.

physical exercises performed on a bicycle cycloergometer increased KYNA and decreased KYN and TRP ${ }^{6}$. Joisten et al. compared the effects of endurance and chronic resistance exercises in healthy adults ${ }^{34}$. They found an enhancement of KYNA and lack of change in KYN content after both exercise interventions; TRP was lowered after resistance but not endurance exercise ${ }^{35}$. The ratio of KYN/TRP and KYNA/KYN was enhanced due to the resistance and endurance activity, respectively. Schlittler et al. showed that endurance training caused an increase in plasma KYNA, while a bout of high-intensity eccentric exercise did not ${ }^{7}$. Taken together, these data confirm that physical activity may affect the metabolism of TRP along the kynurenine pathway; however, the intensity and specific pattern of the alterations differ depending on the nature, extent and duration of the exercise (Table 2). Furthermore, the influence of diet or emotional and mental status has to be taken into account ${ }^{10,17,38,39}$.

Nowadays, the pathophysiological relevance of the impact of physical activity exerted on the kynurenine pathway is a matter of debate ${ }^{16}$. On the one hand, the muscle-mediated shift in the balance between KYN and KYNA in blood reduces KYN availability in the brain, and therefore may beneficially affect central nervous 
system functions ${ }^{9,10}$. On the other hand, the influence of kynurenines on skeletal muscle and peripheral organ should be carefully considered.

Kaiser et al., (2019) presented the hypothesis that activation of AhR by circulating KYN plays a role in muscle loss with aging ${ }^{44}$; indeed, $\mathrm{KYN}$ is an agonist of $\mathrm{AhR}^{11}$. Experimental data revealed that $\mathrm{KYN}$ administration in vivo reduced muscle size and strength in young mice ${ }^{45}$.

The role of KYNA in skeletal muscle morphology and metabolism is unknown. There is only one report showing that KYNA induced protein synthesis and increased the muscle fibres size of C2C12 myoblast cells in vitro. Moreover, KYNA administered in drinking water decreased the loss of muscle mass evoked by limb immobilization in muscle atrophy model in mice ${ }^{46}$. These results were announced in a patent application and not published in a regular peer-reviewed scientific paper.

Unlike in previous studies, we measured the content of TRP, KYN and KYNA in sweat, but not in the blood, because the collection of blood required specialized equipment and assistance of professional personnel. Moreover, it is a painful procedure which is not accepted by many people. Since sweat collection is a non-invasive, painless task, we decided to check its applicability in the determination of kynurenine pathway metabolites during physical activity. Two methods of sweat collection were utilized. A commercial absorbent patch was fixed to the skin (Method A) and commercial ear tips with cotton (Method B) were used. The advantage of Method A is that the patch absorbs sweat throughout the entire test, whereas in the Method B, the drop of sweat was collected at the end of each exercise period, thereby representing temporary sweat content. It is noteworthy that the results obtained by both methods were similar, at least when considering the direction and magnitude of changes in sweat composition. Moreover, the results obtained correspond well with data obtained by other researchers investigating changes of kynurenines in blood ${ }^{6,34,40-43,47}$. Therefore there is reason to conclude that the measurement of TRP, KYN and KYNA in sweat may have the diagnostic potential, and may also help to establish an exercise regime appropriate for the age, gender, and health status of rehabilitation participant. Thanks to the simplicity of the collection of sweat, this procedure may be used to control physical activity on a daily basis according to the strategy of personalized medicine.

The limitation of the present study is that the effect of menstrual cycle was not addressed although number of females exceeded that of males. This issue deserves further detailed analysis, since Hrboticky et al., 1989 reported fluctuation of plasma and urine content of KYN during menstrual period in women orally administered with tryptophan. Interestingly, KYN level was lower in follicular than in luteal phase ${ }^{48}$. This does not correlate to the finding that estrogen $(\mathrm{s})$ increased indoleamine 2,3-dioxygenase and inhibited kynurenine aminotransferases ${ }^{49,50}$.

The another limitation is the absence of a control group and lack of direct correlation between the amount of analyzed compounds in sweat and blood. The control group was not structured. Instead content of tryptophan and kynurenines was individually defined for each participant before the start of exercise cycle and all subsequent measurements were compared to values obtained on day 1. Finally, it should be stressed that our results correlate with data obtained in blood samples by other researchers ${ }^{6,34,40-43,47}$ (Table 2). Evidence is provided that substantial changes in KYN and KYNA content in sweat recorded on day 14 declined or disappeared on day 28 of the exercises. The reason for the dynamics of this phenomenon is not known. It is noteworthy that Millischer et al., 2017, reported unchanged plasma content of KYN and KYNA measured within 1 week after termination of training lasting for 12-weeks. Unfortunately, in this study the content of kynurenines was not determined during the exercises ${ }^{51}$. Certainly, this issue seems to be important because the obtained results may indicate the need for modification of the length, intensity, or set of exercises. It is believed that the use of devices enabling comfortable, non-invasive sweat collection and methods of adequate measurement of kynurenines in secrete can allow easy control and adjustment of rehabilitation processes to individual patient needs.

\section{Conclusions and practical implications}

- TRP, KYN and KYNA is present in detectable amounts in human sweat.

- A two-week cycle of physical exercise decreased the KYN and increased KYNA content in sweat.

- Analysis of the KYN and KYNA sweat content can be considered a useful non-invasive method for monitoring the kynurenine pathway activity during physical training and rehabilitation.

Received: 5 December 2020; Accepted: 13 May 2021

Published online: 27 May 2021

\section{References}

1. Badawy, A.A.-B. Tryptophan availability for kynurenine pathway metabolism across the life span: Control mechanisms and focus on aging, exercise, diet and nutritional supplements. Neuropharmacology 112, 248-263 (2017).

2. Badawy, A.A.-B. Kynurenine pathway of tryptophan metabolism: regulatory and functional aspects. Int. J. Tryptophan Res. IJTR 10, $1178646917691938(2017)$.

3. Fejes, A., Párdutz, Á., Toldi, J. \& Vécsei, L. Kynurenine metabolites and migraine: Experimental studies and therapeutic perspectives. Curr. Neuropharmacol. 9, 376-387 (2011).

4. Vamos, E., Pardutz, A., Klivenyi, P., Toldi, J. \& Vecsei, L. The role of kynurenines in disorders of the central nervous system: Possibilities for neuroprotection. J. Neurol. Sci. 283, 21-27 (2009).

5. Agudelo, L. Z. et al. Skeletal muscle PGC-1 1 modulates kynurenine metabolism and mediates resilience to stress-induced depression. Cell 159, 33-45 (2014).

6. Mudry, J. M. et al. Direct effects of exercise on kynurenine metabolism in people with normal glucose tolerance or type 2 diabetes. Diabetes Metab. Res. Rev. 32, 754-761 (2016).

7. Schlittler, M. et al. Endurance exercise increases skeletal muscle kynurenine aminotransferases and plasma kynurenic acid in humans. Am. J. Physiol. - Cell Physiol. 310, C836 (2016). 
8. Agudelo, L. Z. et al. Skeletal muscle PGC-1 1 1 reroutes kynurenine metabolism to increase energy efficiency and fatigue-resistance. Nat. Commun. 10, 2767 (2019).

9. Notarangelo, F. M., Pocivavsek, A. \& Schwarcz, R. Exercise your kynurenines to fight depression. Trends Neurosci. 41, 491-493 (2018).

10. Pedersen, B. K. Physical activity and muscle-brain crosstalk. Nat. Rev. Endocrinol. 15, 383-392 (2019).

11. Opitz, C. A. et al. An endogenous tumour-promoting ligand of the human aryl hydrocarbon receptor. Nature 478, 197-203 (2011).

12. DiNatale, B. C. et al. Kynurenic acid is a potent endogenous aryl hydrocarbon receptor ligand that synergistically induces interleukin-6 in the presence of inflammatory signaling. Toxicol. Sci. Off. J. Soc. Toxicol. 115, 89-97 (2010).

13. Gostner, J. M. et al. Tryptophan metabolism and related pathways in psychoneuroimmunology: The impact of nutrition and lifestyle. Neuropsychobiology 79, 89-99 (2020).

14. Schwarcz, R. \& Stone, T. W. The kynurenine pathway and the brain: Challenges, controversies and promises. Neuropharmacology 112, 237-247 (2017).

15. Savitz, J. The kynurenine pathway: a finger in every pie. Mol. Psychiatry 25, 131-147 (2020).

16. Martin, K. S., Azzolini, M. \& Lira Ruas, J. The kynurenine connection: how exercise shifts muscle tryptophan metabolism and affects energy homeostasis, the immune system, and the brain. Am. J. Physiol.-Cell Physiol. 318, 818-830 (2020).

17. Cervenka, I., Agudelo, L. Z. \& Ruas, J. L. Kynurenines: Tryptophan's metabolites in exercise, inflammation, and mental health. Science 357, 369 (2017).

18. Turski, M. P., Turska, M., Paluszkiewicz, P., Parada-Turska, J. \& Oxenkrug, G. F. Kynurenic Acid in the Digestive System--New Facts, New Challenges. Int. J. Tryptophan Res. 6, IJTR.S12536 (2013).

19. Fukui, S., Schwarcz, R., Rapoport, S. I., Takada, Y. \& Smith, Q. R. Blood-brain barrier transport of kynurenines: Implications for brain synthesis and metabolism. J. Neurochem. 56, 2007-2017 (1991).

20. Glavin, G. B., Bose, R. \& Pinsky, C. Kynurenic acid protects against gastroduodenal ulceration in mice injected with extracts from poisonous Atlantic shellfish. Prog. Neuropsychopharmacol. Biol. Psychiatry 13, 569-572 (1989).

21. Kaszaki, J. et al. Kynurenic acid inhibits intestinal hypermotility and xanthine oxidase activity during experimental colon obstruction in dogs. Neurogastroenterol. Motil. 20, 53-62 (2008).

22. Varga, G. et al. N-Methyl-D-aspartate receptor antagonism decreases motility and inflammatory activation in the early phase of acute experimental colitis in the rat. Neurogastroenterol. Motil. Off. J. Eur. Gastrointest. Motil. Soc. 22, 217-225 (2010).

23. Bądzyńska, B., Zakrocka, I., Sadowski, J., Turski, W. A. \& Kompanowska-Jezierska, E. Effects of systemic administration of kynurenic acid and glycine on renal haemodynamics and excretion in normotensive and spontaneously hypertensive rats. Eur. J. Pharmacol. 743, 37-41 (2014).

24. Bądzyńska, B. et al. Kynurenic acid selectively reduces heart rate in spontaneously hypertensive rats. Naunyn. Schmiedebergs Arch. Pharmacol. 393, 673-679 (2020).

25. Olenchock, B. A. et al. EGLN1 inhibition and rerouting of a-ketoglutarate suffice for remote ischemic protection. Cell 164, 884-895 (2016).

26. Gosker, H. R., Clarke, G., de Theije, C. C., Cryan, J. F. \& Schols, A. M. W. J. Impaired skeletal muscle kynurenine metabolism in patients with chronic obstructive pulmonary disease. J. Clin. Med. 8, 915 (2019).

27. Wyckelsma, V. L. et al. Kynurenine aminotransferase isoforms display fiber-type specific expression in young and old human skeletal muscle. Exp. Gerontol. 134, 110880 (2020).

28. Kuc, D. et al. Kynurenic acid in human saliva-does it influence oral microflora?. Pharmacol. Rep. PR 58, 393-398 (2006).

29. Buczko, P., Zalewska, A. \& Szarmach, I. Saliva and oxidative stress in oral cavity and in some systemic disorders. J. Physiol. Pharmacol. Off. J. Pol. Physiol. Soc. 66, 3-9 (2015).

30. O'Rourke, L. et al. Tryptophan metabolic profile in term and preterm breast milk: implications for health. J. Nutr. Sci. 7, e13-e13 (2018).

31. Milart, P. et al. Kynurenic acid as the neglected ingredient of commercial baby formulas. Sci. Rep. 9, 6108 (2019).

32. Goldberg, D. P. \& Hillier, V. F. A scaled version of the General Health Questionnaire. Psychol. Med. 9, 139-145 (1979).

33. Zhao, J., Gao, P. \& Zhu, D. Optimization of $\mathrm{Zn} 2+$-containing mobile phase for simultaneous determination of kynurenine, kynurenic acid and tryptophan in human plasma by high performance liquid chromatography. J. Chromatogr. B 878, 603-608 (2010).

34. Joisten, N. et al. Exercise and the Kynurenine pathway: Current state of knowledge and results from a randomized cross-over study comparing acute effects of endurance and resistance training. Exerc. Immunol. Rev. 26, 24-42 (2020).

35. Cui, X. et al. Integrated omics analysis of sweat reveals an aberrant amino acid metabolism pathway in Vogt-Koyanagi-Harada disease. Clin. Exp. Immunol. 200, 250-259 (2020).

36. Baran, H., Kronsteiner, C. \& Kepplinger, B. Kynurenine Aminotransferases I, II and III Are Present in Saliva. Neurosignals 28, $1-13(2020)$.

37. Lewis, G. D. et al. Metabolic signatures of exercise in human plasma. Sci. Transl. Med. 2, 33ra37 (2010).

38. Won, E. \& Kim, Y.-K. Stress, the autonomic nervous system, and the immune-kynurenine pathway in the etiology of depression. Curr. Neuropharmacol. 14, 665-673 (2016).

39. Ogyu, K. et al. Kynurenine pathway in depression: A systematic review and meta-analysis. Neurosci. Biobehav. Rev. 90, 16-25 (2018).

40. Ito, Y. et al. Kynurenine concentration of serum was increased by exercise. Adv. Exp. Med. Biol. 467, 717-722 (1999).

41. Strasser, B. et al. Probiotic supplements beneficially affect tryptophan-kynurenine metabolism and reduce the incidence of upper respiratory tract infections in trained athletes: A randomized, double-blinded, placebo-controlled trial. Nutrients 8, 752 (2016).

42. Strasser, B. et al. Effects of exhaustive aerobic exercise on tryptophan-kynurenine metabolism in trained athletes. PLoS ONE 11, e0153617 (2016).

43. Schlittler, M. et al. Endurance exercise increases skeletal muscle kynurenine aminotransferases and plasma kynurenic acid in humans. Am. J. Physiol. Cell Physiol. 310, C836-840 (2016).

44. Kaiser, H., Parker, E. \& Hamrick, M. W. Kynurenine signaling through the aryl hydrocarbon receptor: Implications for aging and healthspan. Exp. Gerontol. 130, 110797 (2020).

45. Kaiser, H. et al. Kynurenine, a tryptophan metabolite that increases with age, induces muscle atrophy and lipid peroxidation. Oxid. Med. Cell. Longev. 2019, 9894238 (2019).

46. Raynal, S. et al. Use of tryptophan metabolites for treating muscle atrophy. FR3055548A1 (2018).

47. Herrstedt, A. et al. Exercise-mediated improvement of depression in patients with gastro-esophageal junction cancer is linked to kynurenine metabolism. Acta Oncol. Stockh. Swed. 58, 579-587 (2019).

48. Hrboticky, N., Leiter, L. A. \& Anderson, G. H. Menstrual cycle effects on the metabolism of tryptophan loads. Am. J. Clin. Nutr. 50, 46-52 (1989).

49. Jayawickrama, G. S., Nematollahi, A., Sun, G., Gorrell, M. D. \& Church, W. B. Inhibition of human kynurenine aminotransferase isozymes by estrogen and its derivatives. Sci. Rep. 7, 17559 (2017).

50. Li, S. et al. Estrogen induces indoleamine 2,3-dioxygenase expression via suppressors of cytokine signaling 3 in the chorionic villi and decidua of women in early pregnancy,. Am. J. Reprod. Immunol. 83, e13197 (2020).

51. Millischer, V., Erhardt, S., Ekblom, Ö., Forsell, Y. \& Lavebratt, C. Twelve-week physical exercise does not have a long-lasting effect on kynurenines in plasma of depressed patients. Neuropsychiatr. Dis. Treat. 13, 967-972 (2017). 


\section{Author contributions}

Conceptualization, T.S., M. T. and W.A.T..; methodology, T.S., M.T. and T.K.; formal analysis, T.S. and W.A.T..; investigation, T.S., T.K. and M.Z.; resources, T.S., T.K. and M.Z.; data curation, M.T., T.K. and G.Z. writing and original draft preparation, T.S., M.T. and G.Z.; writing, review and editing, T.S.,G.Z..; W.A.T. and P.G. visualization, T.S., M.T., M.Z.; supervision, T.S., W.A.T. and P.G.; project administration, T.S. and P.G ; All authors have read and agreed to the published version of the manuscript.

\section{Competing interests}

\section{The authors declare no competing interests.Additional information}

Correspondence and requests for materials should be addressed to G.Z.

Reprints and permissions information is available at www.nature.com/reprints.

Publisher's note Springer Nature remains neutral with regard to jurisdictional claims in published maps and institutional affiliations.

(c) (i) Open Access This article is licensed under a Creative Commons Attribution 4.0 International License, which permits use, sharing, adaptation, distribution and reproduction in any medium or format, as long as you give appropriate credit to the original author(s) and the source, provide a link to the Creative Commons licence, and indicate if changes were made. The images or other third party material in this article are included in the article's Creative Commons licence, unless indicated otherwise in a credit line to the material. If material is not included in the article's Creative Commons licence and your intended use is not permitted by statutory regulation or exceeds the permitted use, you will need to obtain permission directly from the copyright holder. To view a copy of this licence, visit http://creativecommons.org/licenses/by/4.0/.

(C) The Author(s) 2021 